\title{
Repetition blindness: The effects of stimulus modality and spatial displacement
}

\author{
NANCY KANWISHER \\ University of California, Berkeley, California
}

and

MARY C. POTTER

Massachusetts Institute of Technology, Cambridge, Massachusetts

\begin{abstract}
Repetition blindness (Kanwisher, 1986, 1987) is the failure to detect repetitions of words in lists presented in rapid serial visual presentation (RSVP). Two questions were investigated in the present study. First, if repetition blindness is not found with auditory presentation, it would support a specifically visual account of the effect. Second, if displacement of the two instances in visual space eliminates repetition blindness, it would suggest that repetition blindness is restricted to instances in which identical stimuli are distinguished solely by temporal differences. In Experiment 1, the subjects omitted second occurrences of repeated words in verbatim recall of rapid sentences presented visually (in RSVP), but not auditorily (using compressed speech), indicating that repetition blindness is a modality-specific phenomenon. In Experiments 2 and 3 , repetition blindness was observed even when two occurrences of a written word were presented in different locations, showing that distinct locations do not guarantee token individuation. The results are discussed within a model that distinguishes between processes of type recognition and token individuation.
\end{abstract}

In a recently discovered phenomenon called repetition blindness (Kanwisher, 1986, 1987), viewers fail to report repetitions in lists of words presented in rapid serial visual presentation (RSVP). The failure occurs both in detection tasks in which subjects are asked to report the repeated word, and in immediate recall tasks in which subjects are asked to report all the words, including repetitions. The phenomenon is highly robust for rapid presentation rates (six words/second or faster), and occurs even when the repeated words are separated by one to three intervening words. Furthermore, when repeated words are embedded in sentences that are presented for verbatim recall, the subjects omit the second occurrence even at the sacrifice of sentence meaning and grammaticality. Finally, repetition blindness does not depend on physical identity; the second of the two words is hard to detect even if one occurrence appears in uppercase and the other in lowercase.

Repetition blindness has been interpreted in terms of a distinction between type recognition and token individuation. In recognition, a word is identified as a type (e.g., the word chair). In individuation, an item is character-

\footnotetext{
We thank Audra Noel for research assistance, Arthur Wingfield for the use of a speech compressor, Linda Lombardi for showing us how to use it, and Sue Lima, John Rubin, David Irwin, Richard Held, Anne Treisman, and two anonymous reviewers for helpful comments on the manuscript. This work was supported by NSF Grants BNS83-18156 and BNS86-19053 to Mary C. Potter, and by the MIT Center for Cognitive Science. Requests for reprints may be sent to Nancy Kanwisher, who is now at the Department of Psychology, Tolman Hall, University of California, Berkeley, CA 94720
}

ized as a particular token of a given type (e.g., as the second instance of the word chair). Kanwisher $(1986,1987)$ has argued that repetition blindness occurs because the second instance of a repeated word is not individuated as a distinct token, even though it is recognized as a type. Although the first occurrence interferes with awareness that there was a second occurrence, there is evidence that recognition (type activation) of the second occurrence is not blocked. For example, threshold recognition of the last word in an RSVP list is helped, not hindered, by a prior occurrence of that word in the same list (Kanwisher, $1986,1987)$.

A general model of type and token processing is shown in Figure 1. According to this model, as a word list is presented and new words are recognized, their type nodes become activated. A key feature of the model is that type and token information are encoded in distinct domains. Accordingly, pointers must be established between activated type nodes and token nodes, where the token nodes encode serial order and other kinds of episodic information. The assignment of pointers from types to tokens is what we refer to as token individuation.

A critical feature of the model is a refractory period for token individuation, but not for type activation. That is, following the creation of a pointer from a given type to a token node, there is a period during which no other pointer can originate from that type node. This refractory period, which is a function of both elapsed time and the number of intervening items, does not prevent further activation of the type node; it only inhibits the assignment of a second token to the same type. Thus, when 


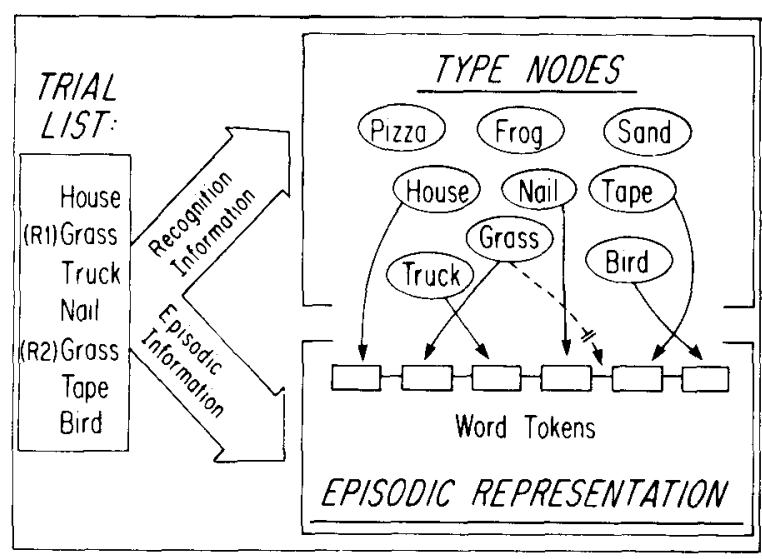

Figure 1. A model depicting the integration of type and token information in the processing of visually presented lists. (From "Repetition Blindness: Type Recognition Without Token Individuation" by N. G. Kanwisher, 1987, Cognition, 27, p. 133. Copyright 1987 by Elsevier Sequoia $S$. A. Reprinted by permission.)

the second occurrence $(\mathrm{R} 2)$ is presented shortly after the first occurrence (R1), its type node will be further activated, but there will be no link to a second token. When this happens, $R 2$ will not be encoded as a new event, but the added type activation it gives rise to will become assimilated to the representation of $\mathrm{R} 1$.

It has been suggested that repetition blindness results not from a particular characteristic of visual word processing, but rather from a general dissociation of type and token processing in vision (Kanwisher, 1986, 1987). Indeed, several recently reported phenomena appear to provide additional cases of repetition blindness not only for words (Humphreys, Besner, \& Quinlan, 1988; Marohn \& Hochhaus, 1988), but also for letters (Mozer, in press). A variety of other visual phenomena could be interpreted in terms of the dissociation between type and token processing. For example, just as we have argued that repetition blindness results from an erroneous assignment of two events to the same token event, Treisman and Schmidt's (1982) "illusory conjunctions" could be considered as erroneous assignments of two visual features from different objects to the same token object. The dissociation of type recognition (e.g., which word or object features were present) and token individuation (e.g., how many instances there were of a word, or which feature went with which object) may also play a role in explaining other visual phenomena, such as iconic memory (Coltheart, 1980; Irwin \& Yeomans, 1986), visual dissociation (Intraub, 1985), visual search (Treisman \& Gelade, 1980 ), and the integration of information across eye movements (Irwin, Brown, \& Sun, 1988; Rayner, McConkie, \& Zola, 1980).

However, before this general interpretation of repetition blindness is adopted, several issues must be addressed. First, is repetition blindness a visual phenomenon, or is it a lexical phenomenon associated with word identification in both visual and auditory modalities? Second, does repetition blindness constitute a specific difficulty in using temporal tags to individuate like tokens when no other individuating features are present?

Concerning the first question, there is evidence that repetition blindness is not restricted to sequences of words. Kanwisher and Potter (1989) have shown that it is difficult to see a repeated letter in a serially presented list of letters (see also Mozer, in press), and pilot evidence suggests that detection of repeated colors is difficult in serially presented lists of colored squares. ${ }^{1}$ Experiment 1 addressed the question of whether repetition blindness generalizes to spoken words. Compressed speech was used to present rapid sentences auditorily. If the limitations on token individuation are a particular characteristic of visual processing, one would expect no decrement in recall for repeated (as compared to unrepeated) words presented auditorily. If problems with repetition arise at an abstract, lexical level, then one would expect to observe repetition deafness.

Experiments 2 and 3 tested whether repetition blindness would be eliminated if the two instances were displaced in space as well as time. Location has been proposed by some researchers (Nissen, 1985; Treisman \& Schmidt, 1982; Ullman, 1984) as the key feature by which an item in a visual array can be specified, or indexed, so one might expect that distinct location information would be necessary and also sufficient to distinguish between different visual tokens of the same type.

\section{EXPERIMENT 1}

In Experiment 1, the subjects were asked to recall whole sentences that contained repeated words. Earlier work (Kanwisher, 1986, 1987) indicated that subjects fail to report second instances of repeated words, even when this leads them to sacrifice the syntax and meaning of the sentence. However, those studies used written words. The present experiment addressed the question of whether repetition deafness would be found for sentences presented auditorily at a similar rate.

To present speech at a rate comparable to that of the previous visual experiments, a speech compressor was used. Time compression of tape-recorded speech increased speech content per unit time while still keeping intelligibility relatively high (see Wingfield, 1975). The sampling method for compression, in which small segments of the recorded message were deleted periodically, was used in Experiment 1. This method resulted in recorded speech that was faster than normal, but not significantly distorted in pitch or quality.

Modality of presentation was a between-subjects variable. The experiment included one repetition condition and two control conditions, as illustrated in Table 1 . In

Table 1

Sample Stimulus Items in Each Condition of Experiment 1

Repeated: When she spilled the ink there was ink all over.

Unrepeated: When she spilled the liquid there was ink all over. Blank: When she spilled the ink there was all over. 
the unrepeated control condition, $\mathrm{R} 1$ was replaced by another word that was acceptable in the sentence. In previous work, it was R2 that subjects failed to report when $\mathrm{R} 1$ was the same word. In the blank control condition, R2 was omitted, leaving an ungrammatical or otherwise anomalous sentence. One purpose of this control was to assess the propensity to intrude $\mathrm{R} 2$ even if it was not perceived; a second purpose was to encourage subjects to report what they heard or saw, whether or not it was a grammatical sentence.

\section{Method}

Subjects. Eighteen volunteer subjects participated in the experiment, 9 in the auditory group and 9 in the visual group. All were native speakers of American English and were paid for their participation.

Materials. Nine sentences containing repeated words were written. In one control version of each sentence, the first occurrence of the critical word (RI) was replaced by another word, often a synonym, that preserved the general structure and meaning of the sentence (the unrepeated control). In the other control version, the second occurrence (R2) was omitted (the blank control); the resulting sentence was usually ungrammatical. Thus, each sentence had three versions: one with repeated critical words, one with unrepeated critical words, and one with a blank in place of R2. The critical word pairs never included the first or last word of the sentence, and there were from one to three intervening words. Although some of the sentences were slightly awkward, all except those in the blank version were grammatically acceptable. The blank version was always ungrammatical or anomalous. The nine sentences and their controls are shown in Appendix A, and examples are shown in Table 1.

There were 12 additional filler sentences in Experiment 1, used to study a different question. These fillers, which included some pairs of similarly spelled words but no identically repeated ones, were interspersed randomly with the test sentences.

Design. Except for modality (visual vs. auditory), the design was the same for both groups. The three versions of each sentence (repeated, unrepeated, and blank) appeared in different versions of the experiment, counterbalanced so that each subject saw or heard three sentences in each condition for a total of nine test sentences per subject.

Procedure. For the visual version of the experiment, each trial began when the subject pressed the space bar on the computer keyboard. A row of asterisks appeared for $750 \mathrm{msec}$ in the center of the screen at the same location as the subsequent words. Next, the sentence appeared one word at a time in the same place. Except for the first letter of the first word, all words were in lowercase. Each word was left-justified and was displayed for $117 \mathrm{msec}$.

The subjects were instructed to read the sentence and recall it aloud as soon as it ended. They were warned that some sentences would be strange or ungrammatical, and that they were to repeat them "as is," without "fixing them up."

For the auditory version, the speech compressor was used to increase the presentation rate of spoken sentences to about 7.5 words per second (an average of $133 \mathrm{msec}$ per word). This was similar to the rate used in the corresponding visual version of this experiment, and identical to the presentation rate used in several earlier visual experiments that demonstrated marked repetition blindness (Kanwisher, 1986). Because parametric studies of compressed speech (Wingfield \& Nolan, 1980) have suggested that understanding would be somewhat less robust at rapid presentation rates than would understanding at equivalent rates of RSVP, the slightly slower rate of auditory presentation was considered acceptable in Experiment 1.

The subjects in the auditory version were instructed to listen to the sentence as carefully as possible, and to repeat it as soon as it ended. They were warned that it would be difficult to hear, and that some of the sentences would be strange or ungrammatical, but that they should still try to repeat them exactly as they heard them. Each trial began when the subject pushed the "play" button on the tape recorder. After a pause, the words "Trial number $x$ " were spoken normally, followed by the compressed sentence. After each sentence, the subject stopped the tape recorder and recalled the sentence aloud. For both the auditory and the visual versions of the experiment, three practice trials without repetitions preceded the test trials.

Apparatus. The visual version of Experiment 1 was run on a Terak microcomputer with a rapid-fade phosphor. The auditory version was run on a double-track Sony tape recorder. The compressed tape was made on an electromechanical compressor of the Fairbanks type.

\section{Results}

Overall, recall accuracy for the sentences was high for both visual and auditory versions of the experiment. The number of trials in which the subjects included $R 1$ and R2 in their recall of the sentence was scored. When only one of the critical words was recalled, it was almost always apparent from its serial position whether it was $R 1$ or R2. Whenever there was any question about this, the item was scored conservatively as an inclusion of R2.

The primary focus of Experiment 1 was on recall of $\mathrm{R} 2$. The subjects in the visual version recalled $\mathrm{R} 2$ in $30 \%$ of repeated trials, $93 \%$ of unrepeated trials, and $0 \%$ of blank trials (i.e., R2 was never intruded). The subjects in the auditory version recalled R2 in $100 \%$ of repeated trials, $89 \%$ of unrepeated trials, and $0 \%$ of blank trials. An analysis of variance by subjects and items was carried out on the number of correct recalls of $R 2$ in the repeated and unrepeated conditions, omitting the blank condition. There were significant main effects of repeatedness $\left[F_{1}(1,16)=16, M S_{\mathrm{e}}=5.4, p<.001 ; F_{2}(1,8)\right.$ $=28, p<.001]$, modality $\left[F_{1}(1,16)=29, M S_{\mathrm{e}}=9.0\right.$, $\left.p<.001 ; F_{2}(1,8)=24, p<.001\right]$, and a significant interaction of repeatedness $\times$ modality $\left[F_{1}(1,16)=33\right.$, $\left.M S_{\mathrm{e}}=11, p<.001 ; F_{2}(1,8)=31, p<.001\right]$. As Table 2 shows, repetition blindness was marked in the visual version and nonexistent in the auditory version.

The percentages of recalls of $\mathrm{R} 1$ and $\mathrm{R} 2$ in each condition are shown in Table 2 . An analysis of variance by subjects was carried out on recall of $\mathrm{R} 1$. There were no significant main effects or interactions (all $F_{\mathrm{s}}<1$ ), supporting the assumption that auditory and visual conditions were well matched for intelligibility and comprehension.

\section{Discussion}

Repetition blindness occurred with visually presented sentences, but did not occur with spoken sentences presented at a similar rate. Because performance in the unrepeated condition was less than $100 \%$ and was similar for the auditory and visual groups, it is unlikely that the task was simply easier overall for the auditory group. Hence, repetition blindness appears to be a modalityspecific phenomenon. Either it occurs at a stage of processing before visual and auditory linguistic inputs con- 
Table 2

Percent Recall of $\mathrm{R} 1$ and $\mathrm{R} 2$ in Each Condition of Experiment 1

\begin{tabular}{lccccccc}
\hline & \multicolumn{3}{c}{ R1 } & & \multicolumn{3}{c}{ R2 } \\
\cline { 3 - 4 } \cline { 5 - 7 } Condition & Repeated & Unrepeated & Blank & & Repeated & Unrepeated & Blank* \\
\hline Visual & 96 & 88 & 88 & 30 & 93 & 0 \\
Auditory & 88 & 88 & 92 & & 100 & 89 & 0 \\
\hline
\end{tabular}

${ }^{*}$ Recall of R2 was an intrusion, because R2 was omitted in the stimulus sentence.

verge, or it occurs at a later stage but is affected by earlier modality-specific information. For example, a precategorical acoustic buffer might store the auditory stimulus string while it is being presented. Such a buffer might then pass the information along at a rate slow enough to permit token individuation at the hypothesized later stage. Estimates of the size of the precategorical buffer (see, e.g., Crowder, 1976) make this account unlikely. Alternatively, a phonological buffer, such as that described by Baddeley (1986), might permit a slower rate of token assignment. Yntema, Wozencraft, and Klem (1964) have shown, however, that listeners are unable to store a rapid string of compressed digits, suggesting that the phonological buffer would be insufficient as a temporary store for words not yet assigned a token representation. Thus, it seems more likely that repetition blindness occurs at an early stage in visual processing, before visual and auditory linguistic inputs converge.

Another reason to suspect that repetition blindness results from processes particular to vision is that, as Kubovy (1988) has claimed, auditory processing is inherently temporal and visual processing is not (however, see Handel, 1988, for a contrary view). Earlier work on temporal numerosity (Lechelt, 1975) showed that subjects can count the number of repeated auditory events presented in a rapid sequence better than they can count the number of repeated visual events in a rapid sequence. Many kinds of temporal distinctions, though not all, ${ }^{2}$ are encoded at a finer level of detail for auditory presentations than they are for visual presentations (Glenberg \& Swanson, 1986).

\section{EXPERIMENT 2}

Experiment 2 addressed a second question about repetition blindness. If, as Experiment 1 suggested, it is a purely visual phenomenon, then one might expect it to be sensitive to parameters (e.g., spatial location) that have been suggested to play a key role in visual information processing. Treisman and Gelade (1980) have argued that visual features are detected in an array before they are localized. Attention must be directed to the location of a particular item, in order to conjoin its different features. The location of an array item thus serves to index featural information about that item (Nissen, 1985). Similarly, Ullman (1984) has suggested that the visual system might use an operation called marking during visual search to tag the locations that have already been the focus of processing, in order to distinguish them from locations that have not yet been visited.

These investigators and others concur in proposing that location is used to specify particular items, or tokens, and information about item identities, or types, can be indexed under those locations. If this is a general strategy of the visual system, it suggests that repetition blindness may occur only when the viewer must depend solely on temporal differences to distinguish between repeated items. One would then expect word repetitions to be readily detected if the two occurrences were displayed in different locations. This possibility was tested in Experiment 2 by presenting RSVP sentences in which the words were staggered across the screen from left to right.

\section{Method}

Subjects. Twenty-four new subjects from the previously described pool took part, 12 in the stationary group and 12 in the moving group.

Materials and Design. Thirty test sentences were written (see Appendix B). They contained repeated words and were much like the sentences in Experiment 1. There were usually two words between the two occurrences of the repeated word, and, in many cases, the repeated word was required for grammaticality. Eleven filler sentences without repetitions were included in the experiment to provide more variety in sentence structure. As in Experiment 1 , each test sentence occurred in each of three conditions, counterbalanced across subjects: repeated, unrepeated, and blank. There were three practice sentences without repetitions.

The format-moving or stationary-was a between-subjects variable. In the stationary condition, sentences were presented as in Experiment 1, with every word appearing at the same location. In the moving condition, the sentence words were displayed serially, but the beginning of each word appeared two character spaces (i.e., $.5^{\circ}-.75^{\circ}$ ) to the right of the beginning of the previous word. Thus, successive words overlapped but were staggered from left to right as they appeared. As with the RSVP condition, the previous word disappeared as the next word appeared. The spatial positions of successive words overlapped, making it possible for the subject to read at a high rate without requiring an eye movement for each word. On $80 \%$ of trials, R1 and R2 did not spatially overlap at all, because they were separated by two or more staggered intervening words. To make the different spatial position of each word more apparent, a horizontal rectangle enclosed the entire space to be occupied by the sentence, remaining in view while the sentence was displayed.

At the beginning of each trial, a row of asterisks appeared. They were positioned inside the rectangle on the left side, for the moving condition, or in the center of the screen, for the stationary condition. For both moving and stationary sentences, each word was displayed for $117 \mathrm{msec}$.

Procedure. The procedure was identical to the visual condition of Experiment 1, except that the subjects in the moving condition 
Table 3

Percent Recall of R1 and R2 in Each Condition of Experiment 2

\begin{tabular}{lccccccc}
\hline \multirow{2}{*}{$\begin{array}{l}\text { Viewing } \\
\text { Condition }\end{array}$} & \multicolumn{3}{c}{$\mathrm{R} 1$} & & \multicolumn{3}{c}{$\mathrm{R} 2$} \\
\cline { 2 - 4 } \cline { 6 - 8 } & Repeated & Unrepeated & Blank & & Repeated & Unrepeated & Blank* \\
\hline Stationary & 82 & 82 & 92 & & 37 & 70 & 7 \\
Moving & 85 & 81 & 82 & & 38 & 75 & 2 \\
\hline
\end{tabular}

*Recall of R2 was an intrusion, because R2 was omitted in the stimulus sentence.

were told that the sentence would be moving to the right inside a horizontal rectangle.

\section{Results and Discussion}

The percentage of correct inclusions of $\mathrm{R} 1$ and $\mathrm{R} 2$ are shown in Table 3 . An analysis of the $\mathrm{R} 1$ responses showed no significant effects, with all $F \mathrm{~s}<1$. Thus, perception of nonrepeated words was not significantly different for the moving and stationary sequences. In the analysis of $\mathbf{R} 2$ responses, $\mathbf{R} 2$ was included in recall less often in the repeated trials $(37 \%)$ than it was in the unrepeated trials $(72 \%)\left[F_{1}(1,22)=58, M S_{\mathrm{e}}=147, p<.001 ; F_{2}(1,28)\right.$ $=56, p<.001]$. There was no significant main effect of format, and, in particular, no significant interaction of repeatedness $\times$ format (both $F_{1}$ and $F_{2}<1.0$ ).

Thus, providing a spatial distinction between R1 and $\mathrm{R} 2$ did not reduce repetition blindness. Token individuation apparently fails at rapid rates whether the tokens are separated spatially or not.

Two possible counterarguments should be considered, however. First, it is possible that the two locations in the moving condition were not perceptually discriminable. This is unlikely, however, because a conspicuous reference frame was provided for the subjects in the form of a rectangle surrounding the sentence; as the sentence was presented, the words moved very obviously from the left to the right side of the rectangle. The first and second occurrences of the repeated word were staggered either four, six, or eight character spaces apart, and usually the two words had no spatial overlap. Finally, on the scale of the visual discriminations required to read letters in the words, R1 and R2 were very far apart. ${ }^{3}$

A second counterargument is that $\mathrm{R} 1$ and $\mathrm{R} 2$ may have fallen on the same or very nearby locations in a retinal coordinate system, because of eye movements following the successive words. It is unclear what type of coordinate system might be used for tagging recognized items by location, and retinal coordinates are at least a possibility. Had R2 fallen on the same retinal location as R1, the effect might have been equivalent to that in stationary RSVP. It is unlikely, however, that only retinal coordinates are used to tag items, or the viewers would have difficulty determining token identity across fixations, which they certainly can do in normal viewing. In any case, because eye movements could not have occurred in perfect synchrony with the shifting words, at least some of the time R1 and R2 would have appeared at different retinal locations, and yet there was no reduction in repetition blindness in this condition.
Thus, neither of the counterarguments is very persuasive, and it seems safe to conclude that spatial separation between $\mathrm{R} 1$ and $\mathrm{R} 2$ does not automatically attenuate repetition blindness. However, it is still possible that a more obvious spatial separation of R1 and R2 would be sufficient to diminish blindness, and that possibility was investigated in Experiment 3.

\section{EXPERIMENT 3}

In Experiment 2, the moving condition was chosen so that successive words (and, hence, R1 and R2) were as far apart as they could be without hindering overall reading ability. It could be argued, however, that the location cues provided in the moving condition were of limited use, because they were lost in the context of the continuous rightward progression of the words. If so, location cues might be enhanced if there were only two distinct locations in which the sentence words could appear. In Experiment 3, this possibility was tested by having the first half of the sentence appear in one location, and the second half in a new location immediately below the first.

\section{Method}

Subjects. Thirty new subjects from the previously described pool took part, 15 in the stationary group and 15 in the "jumping" group.

Materials and Design. The stimulus materials and design were identical to those used in Experiment 2. Format-stationary or jumping - was a between-subjects variable. The stationary condition was identical to the one used in Experiment 2. In the jumping condition, the first part of the sentence appeared in one position and the remainder in a second position, about $1^{\circ}$ below the first. The jump occurred on the second word after R1, so that R2 appeared below R1. There was no pause between the offset of the last upper word and the onset of the first lower word. Ordinarily R1 was the next to last upper word and R2 was the second lower word. For both stationary and jumping conditions, each word was displayed for $117 \mathrm{msec}$.

Procedure. The procedure was identical to that of Experiment 2, except that the subjects in the jumping condition were told that there would be a downward jump in midsentence, with subsequent words appearing in the new lower location.

\section{Results and Discussion}

The percentages of correct inclusions of $\mathrm{R} 1$ and $\mathrm{R} 2$ are shown in Table 4 . An analysis of the R1 responses showed no significant effects, with all $F$ s $<1$. Thus, perception of nonrepeated words was not significantly different for the jumping and stationary sequences. In the analysis of $R 2$ responses, $R 2$ was included in recall less often in the repeated trials $(44 \%)$ than it was in the unrepeated trials 
Table 4

Percent Recall of R1 and R2 in Each Condition of Experiment 3

\begin{tabular}{|c|c|c|c|c|c|c|}
\hline \multirow{2}{*}{$\begin{array}{l}\text { Viewing } \\
\text { Condition }\end{array}$} & \multicolumn{3}{|c|}{$\mathrm{R} 1$} & \multicolumn{3}{|c|}{$\mathrm{R} 2$} \\
\hline & Repeated & Unrepeated & Blank & Repeated & Unrepeated & Blank* \\
\hline Stationary & 87 & 90 & 87 & 41 & 75 & 3 \\
\hline Jumping & 91 & 91 & 87 & 47 & 69 & 4 \\
\hline
\end{tabular}

*Recall of R2 was an intrusion, because R2 was omitted in the stimulus sentence.

$(72 \%)\left[F_{1}(1,28)=81, M S_{\mathrm{e}}=115, p<.001 ; F_{2}(1,28)\right.$ $=50, p<.001]$. There was no significant main effect of format $\left[F_{1}\right.$ and $\left.F_{2}(1,28)<1\right]$.

However, there was a marginally significant interaction of repeatedness $\times$ format $\left[F_{1}(1,28)=4, M S_{\mathrm{e}}=6\right.$, $\left.p=.05 ; F_{2}(1,28)=3.9, p=.06\right]$. A separate analysis of the jumping condition showed that the repeatedness effect was significant for this group alone $\left[F_{1}(1,14)=\right.$ $\left.21.85, p<.001 ; F_{2}(1,28)=14, p<.001\right]$.

In other words, although the jumping condition somewhat diminished the severity of repetition blindness, a strong and highly significant effect remained. Thus, even a very clear spatial separation between $R 1$ and $R 2$ is not sufficient to eliminate repetition blindness.

Although enhanced location cues seem to diminish repetition blindness, the source of the effect is not clear. The jumping condition confounds enhanced location cues with other factors. In particular, the jump provides a clear episodic marker in the middle of the sentence that subjectively partitions the words of the sentence into two groups, and it may be this grouping effect, rather than the two locations themselves, that helps to individuate $R 1$ and $R 2$. If this hypothesis is correct, one would expect repetition blindness to be reduced by other visual events that make a break between $R 1$ and $R 2$, even if they remain in the same location. This hypothesis will be followed up in future research.

Whatever the explanation for the diminished effect of repetition in Experiment 3, the main conclusion is that repetition blindness remains robust, even with a conspicuous shift in spatial location. This agrees with Mozer's (in press) findings with single, simultaneous arrays of letters; when subjects were asked how many letters were in the array, they gave lower estimates for arrays containing repetitions. This suggests that even though the letters are in different locations, repetition impairs perception.

\section{GENERAL DISCUSSION}

The present experiments address two questions about the scope of repetition blindness. In Experiment 1, repetition blindness did not occur for auditory sentences presented at a rate sufficient to produce repetition blindness with visual sentences. Thus, repetition blindness does not seem to be characteristic of word identification and retention in general. Rather, it is restricted to visually presented words, at least at the rate tested.

Experiments 2 and 3 addressed a second question about repetition blindness: whether it occurs only when there is no spatial disparity between the two occurrences of the repeated word. However, the results of Experiments 2 and 3 were not consistent with this account: Different locations did not always enable the subjects to encode R1 and R2 separately. Instead, the difficulty of individuating second tokens appears to be a more general problem that occurs whether or not there are also configural (letter case) or spatial differences between the tokens.

\section{Implications for the Model}

How do these findings fit into the model discussed earlier? Recall that the model distinguishes between the two processes of recognizing an item (in this case, a word) as a given type and individuating the item as a particular token of that type. It was suggested that the second instance of a repeated word is recognized but not tokenindividuated, because a given type can only be assigned to one token within a certain time interval (defined either absolutely or with reference to the number of intervening items). The present experiments provide new evidence about the modality specificity (Experiment 1) and location independence (Experiments 2 and 3) of the phenomenon.

Consider first the result of Experiment 1 that repetition blindness does not occur for auditorily presented sentences. If repetition blindness occurs at a level of processing that encodes abstract lexical entries, one would expect the phenomenon to be independent of the modality of presentation. Because it was not, repetition blindness must reside at a level that encodes only visual presentations. On the other hand, it is not simply configural visual information that is involved, since repetition blindness occurs across changes in letter case (Kanwisher, 1986). The existence of a level of visual representation that encodes abstract letter information independent of case has been demonstrated by Friedman (1980) and Rayner et al. (1980), and it is possible that repetition blindness occurs at a level that represents abstract letters but not words themselves.

Earlier work (Kanwisher, 1986; Kanwisher \& Potter, 1989) has indicated, however, that repetition blindness does not occur at the level of individual letters within a string. In free recall from RSVP word lists, single-letter repetition blindness was not observed when the lists included pairs of words that had one identical letter in the same position. The pairs were chosen so that blindness to the repeated letter would result in a new word. For example, "plant" preceded "heart," inviting a report of "hear" if the repeated " $t$ " had produced blindness. This type of error never occurred. It was concluded that whole words or letter-strings, not single letters, are subject to repetition blindness. 
Although the question of the abstractness of the type nodes is not yet completely answered, upper and lower bounds can be set. The type nodes involved in repetition blindness fall somewhere between a level representing individual letters and one representing abstract (modalitygeneral) lexical entries. A reasonable guess is that repetition blindness occurs at a level that encodes orthographic lexical information.

However, it may be that the level of abstraction of the units that are subject to repetition blindness is a function of the kinds of items presented and encoding performed. Similar to the content of a chunk in short-term memory, the nature of the type involved in repetition blindness may depend both on the stimulus and on the task required of the subject. These issues are explored in Kanwisher and Potter (1989).

What are the implications of Experiments 2 and 3 for the model? The fact that repetition blindness occurs even when $R 1$ and $R 2$ are spatially distinguished indicates that the difficulty of individuating second tokens of a given type cannot be construed narrowly as a problem of temporal individuation alone. Rather, it seems that individuation of a second visual token of the same type is difficult no matter what kinds of distinguishing cues (e.g., letter case or location) are available in addition to temporal difference. The results of Experiment 3 could be taken as evidence that if the location cues become extremely salient, they can somewhat diminish the severity of repetition blindness. But this diminution is far from total, and it could arise from grouping effects that have nothing specifically to do with location.

These findings suggest that the repetition blindness model may generalize to any visual types and to any visual tokens. We therefore hypothesize that repetition blindness reflects not a particular property of the way serially presented word lists are processed, but a general dissociation between the processing of visual types and visual tokens.

If so, repetition blindness may elucidate a number of other visual phenomena in which distinctions must be made between like tokens. Treisman and her colleagues (e.g., Treisman \& Gelade, 1980) have shown, for example, that conjunctive visual search requires serial acts of attention to be directed to particular items in a display in order to conjoin their different features. This process of feature conjunction can be thought of as the assignment of different feature types to single display tokens, which would necessarily entail the indexing, or singling out, of particular tokens. ${ }^{4}$ It remains to be discovered whether token individuation as required for repetition detection is subserved by the same cognitive mechanism as token indexing in conjunctive visual search. Future research will explore the connection between these and other visual phenomena.

\section{REFERENCES}

Baddeley, A. (1986). Working memory. Oxford: Clarendon Press. Broadbent, D. E., \& Broadbent, M. H. P. (1987). From detection to identification: Response to multiple targets in rapid serial visual presentation. Perception \& Psychophysics, 42, 105-113.

Coltheart, M. (1980). Iconic memory and visible persistence. Perception \& Psychophysics, 27, 183-228.

Crowder, R. G. (1976). Principles of learning and memory. Hillsdale, NJ: Erlbaum.

Friedman, R. B. (1980). Identity without form: Abstract representations of letters. Perception \& Psychophysics, 28, 53-60.

Glenberg, A., \& SWANSON, N. (1986). A temporal distinctiveness theory of recency and modality effects. Journal of Experimental Psychology: Learning, Memory, \& Cognition, 12, 3-15.

HANDEL, S. (1988). Space is to time as vision is to audition: Seductive but misleading. Journal of Experimental Psychology: Human Perception \& Performance, 14, 315-317.

Humphreys, G. W., Besner, D., \& Quinlan, P. T. (1988). Event perception and the word repetition effect. Journal of Experimental Psychology: General, 117, 51-67.

INTRAUB, H. (1985). Visual dissociation: An illusory conjunction of pictures and forms. Journal of Experimental Psychology: Human Perception \& Performance, 11, 431-442.

IRWIN, D., BRown, J., \& SUN, J. (1988). Visual masking and visual integration across saccadic eye movements. Journal of Experimental Psychology: General, 117, 276-287.

IRWIN, D., \& Yeomans, J. (1986). Sensory registration and informational persistence. Journal of Experimental Psychology: Human Perception \& Performance, 12, 343-360.

KANWISHER, N. G. (1986). Repetition blindness: Type recognition without token individuation. Unpublished doctoral dissertation, Massachusetts Institute of Technology, Cambridge.

KANWISHER, N. G. (1987). Repetition blindness: Type recognition without token individuation. Cognition, 27, 117-143.

Kanwisher, N. G., \& Potter, M. C. (1989). Repetition blindness: Levels of processing. Manuscript submitted for publication.

KuBovy, M. (1988). Should we resist the seductiveness of the space: time:vision:audition analogy? Journal of Experimental Psychology: Human Perception \& Performance, 14, 318-320.

LECHELT, E. C. (1975). Temporal numerosity discrimination: Intermodal comparisons revisited. British Journal of Psychology, 66, 101-108.

MAROHN, K. M., \& HochHaUs, L. (1988). Different case repetition still leads to perceptual blindness. Bulletin of the Psychonomic Society, 26, 29-31.

MOzer, M. (in press). Types and tokens in visual letter perception. Journal of Experimental Psychology: Human Perception \& Performance.

NisSEN, M. J. (1985). Accessing features and conjunctions: Is location special? In M. I. Posner \& O. S. M. Marin (Eds.), Mechanisms of attention: Attention and performance XI, pp. 205-219, Hillsdale, NJ: Erlbaum.

RAyner, K., McConkie, G. W., \& Zola, D. (1980). Integrating information across eye movements. Cognitive Psychology, 12, 206-226.

Treisman, A., \& Gelade, G. (1980). A feature integration theory of attention. Cognitive Psychology, 12, 97-136.

Treisman, A., \& SCHMIDT, H. (1982). Illusory conjunctions in the perception of objects. Cognitive Psychology, 14, 107-141.

UlLman, S. (1984). Visual routines. Cognition, 18, 97-159.

WingFielD, A. (1975). Acoustic redundancy and the perception of timecompressed speech. Journal of Speech \& Hearing Research, 18, 96-104.

WINGFIELD, A., \& NolaN, K. A. (1980). Spontaneous segmentation in normal and in time-compressed speech. Perception \& Psychophysics, 28, 97-102.

YNtema, D. B., Wozencraft, F. T., \& Klem, L. (1964, October). Immediate serial recall of digits presented at very high rates. Paper presented at the meeting of the Psychonomic Society, Niagara Falls, Ontario.

\section{NOTES}

1. A recent study by Broadbent and Broadbent (1987) demonstrated that subjects perform badly in reporting the second of two (different) 
targets in an RSVP list when the targets are specified as the only items in uppercase. This finding might be explained in terms of repetition blindness for the second occurrence of the capitalization feature (type), although this would not explain why the second of two animal-name targets was also difficult to report. (Is the category animal name a type? Certainly not a visual type.) Their phenomenon seems to have to do with a delay in restarting the target search, after stopping to identify and register the first target. In the present recall experiments, there is no target, and in the repetition-detection procedure used in Kanwisher (1986, 1987), the target is not known until the second word (R2) appears. Thus, the Broadbent and Broadbent result is not directly related to repetition blindness.

2. Visual flicker fusion occurs at a higher frequency than does the fusion of acoustic pulses into an auditory experience of pitch.

3. Furthermore, a recent study by Marohn and Hochhaus (1988) shows that the subjects fail to perceive brief second presentations of words when they are masked and immediately follow a 500 -msec presentation of the same word appearing two lines above the location of the target. This phenomenon, which Marohn and Hochhaus call "perceptual blindness," occurs when the two occurrences are in opposite letter case, and seems to be a variant of repetition blindness. If so, their study shows that location cues that are even clearer than those used in Experiment 2 fail to eliminate repetition blindness.

4. Single-feature search, by contrast, logically requires no token information, and subjects perform it in a fast and parallel manner.

\section{APPENDIX A Stimulus Materials of Experiment 1}

1. The brown couch (sofa) and black couch were stolen.

2. To use (a) radio (the headphones) the radio must have batteries.

3. We asked for water (wine) although water was unavailable (available).

4. When she spilled the ink (liquid) there was ink all over.

5. We got into this van (vehicle) and another van for the commute.

6. His collection of books (things) will include more books about travel.

7. It was work (day) time so work had to get done.

8. Her jacket was red (pink) because red is conspicuous.

9. We were eating (dining) although eating was unnecessary.

Note--The words used in the unrepeated condition are shown in parentheses.

\section{APPENDIX B}

Stimulus Materials of Experiments 2 and 3

1. When the host served rum (them) they drank rum all evening.

2. In the apple (fruit) crate one apple was left.

3. She said her horse (pony) won the horse show.

4. Artists who paint (draw) scenery can't paint people.

5. In general, tall (healthy) parents have tall children.

6. Those cows (animals) are dairy cows I think.

7. The little girl hit the ball (boy) on the ball field.

8. The cherry trees (bushes) and peach trees were blossoming.

9. They wanted to rent boats (canoes) but no boats were available.

10. My friends prefer playing (throwing) frisbee(s) to playing tennis.

11. I hate raw (cold) celery but raw carrots are all right.

12. At the party people (everyone) talked and people danced.

13. She put the pencil (eraser) inside the pencil box.

14. People who read (like) magazines also read books.

15. He loves to play the violin (music) although his violin squeaks.

16. She hid the wine bottles (corks) and other bottles quickly.

17. They were jogging (running) because jogging is healthful.

18. The blue car (truck) and the beige car are new.

19. The children would rather eat (have) nothing than eat spinach.

20. He says that good students (pupils) always are students who think.

21. His collection of paintings (art) includes several paintings of Venice.

22. We prefer (like) Paris but they prefer Boston.

23. She read this novel (book) and another novel on the train.

24. If you ever play (go) they might play also.

25. The drunken hunter (man) shot another hunter by accident.

26. The skilled craftsman makes wooden (brick) houses and wooden boats.

27. Doctors advise against smoking (cigarettes) because smoking causes cancer.

28 . When Joe sleeps (naps) he usually sleeps soundly.

29 . He listens to the radio (it) whenever the radio works.

30. The tailor can sew (mend) dresses or sew pants.

Note-The words used in the unrepeated condition are shown in parentheses. 